\title{
Etude de la supplémentation de la poudre de curcuma comme phytobiotique promoteur de croissance dans l'aliment des poulets au Burkina Faso
}

\author{
Bansé OUEDRAOGO ${ }^{1 *}$, Zara S. NIKIEMA ${ }^{2}$, Jacob SANOU ${ }^{2}$ et Jean Sibiri ZOUNDI ${ }^{3}$ \\ ${ }^{1}$ Département Production Animales / Institut de l'Environnement et de Recherches Agricoles (INERA), 04 BP \\ 8645 Ouagadougou 04, Burkina Faso. \\ ${ }^{2}$ Département Production Végétale / Institut de l'Environnement et de Recherches Agricoles (INERA), 04 BP \\ 8645 Ouagadougou 04, Burkina Faso. \\ ${ }^{3}$ Zootechnicien, Maître de Recherches Institut de l'Environnement et de Recherches Agricoles (INERA) 04 BP \\ 8645 Ouagadougou 04 Département Gestion des Ressources Naturelles et Systèmes de Production (GRN/SP), \\ Burkina Faso. \\ *Auteur correspondant ; E-mail : banse_ouedraogo@yahoo.fr; Tél :00226 70376152
}

\begin{tabular}{ccc}
\hline Received: 18-06-2021 & Accepted: 12-10-2021 & Published: 30-10-2021 \\
\hline
\end{tabular}

\section{RESUME}

La présente étude est une contribution à la recherche d'une voie alternative d'amélioration de l'alimentation avicole au Burkina Faso. L'objectif était d'évaluer les effets de l'incorporation de la poudre de rhizome de curcuma (Curcuma longa) sur les performances de croissance et les caractéristiques de la carcasse des poulets de chair. Pour ce faire une expérience a été conduite pendant huit (08) semaines sur 120 poussins hybrides Bleu d'Hollande répartis au hasard en trois (03) lots de 40 sujets recevant trois (03) traitements alimentaire en croissance-finition (R0, R1, R2) contenant respectivement 0,2 et $4 \%$ de poudre de curcuma. Chaque lot a été subdivisé en deux (02) sous-lots de 20 sujets correspondant à deux (02) répétitions. Les résultats ont montré que les poids vifs moyens ont connu des variations non significatives avec R2 (4\%) $(1084,63 \pm 236,47 \mathrm{~g}), \mathrm{R} 1(2 \%)(1058,54 \pm 235,12 \mathrm{~g})$ et le témoin R0 $(1025,04 \pm 287,55 \mathrm{~g})$. L'indice de consommation des oiseaux, les consommations alimentaires sont restés non significatives par rapport au témoin. Pour des taux d'incorporation de 2 et $4 \%$, la poudre de rhizome de curcuma n'a pas engendré des performances de croissance et de conformation de carcasse significativement différentes par rapport aux sujets témoins. On pourrait conclure que l'utilisation du curcuma comme additif alimentaire est sans effets néfastes sur les performances globales des poulets de chair et une étude de rentabilité devrait être menée. Elle montre que l'incorporation du curcuma à des taux de $2 \%$ et $4 \%$ pourrait réduire les charges en d'autres ingrédients conventionnels couteux comme le soja torréfié dans les rations alimentaires de poulet de chair.

(C) 2021 International Formulae Group. All rights reserved.

Mots clés: Curcuma longa, poulets de chair, performances de croissance, caractéristiques de la carcasse.

\section{Study of the supplementation of turmeric powder as a growth promoting phytobiotic in chicken feed in Burkina Faso}

\begin{abstract}
The objective of this study is to evaluate the effects of incorporation of turmeric Rhizome powder (Curcuma longa) on growth performance and carcass characteristics of broilers. To do this, an experiment was conducted for eight (08) weeks on 120 hybrid Dutch Blue chicks randomly divided into three(03) batches of 40
\end{abstract}


subjects receiving three(3) growth-finishing feed treatments (R0, R1, R2) containing 0,2 and $4 \%$ turmeric powder respectively. Each batch was subdivided into two (02) sub-batches of 20 subjects corresponding to two (02) replicates. The results showed that the mean live weights showed non-significant variations with R2 (4\%) $(1084.63 \pm 236.47 \mathrm{~g}), \mathrm{R} 1(2 \%)(1058.54 \pm 235.12 \mathrm{~g})$ and the control R0 (1025.04 $\pm 287.55 \mathrm{~g})$. The bird consumption index, feed consumption remained insignificant compared to the control. For incorporation rates of $2 \%$ and $4 \%$, turmeric rhizome powder did not result in significantly different growth performance and carcass conformation compared to the control. It could be concluded that the use of turmeric as a feed additive has no adverse effects on the overall performance of broilers and a cost-effectiveness study should be conducted. It shows that the incorporation of turmeric at levels of $2 \%$ and $4 \%$ could reduce the loadings of other expensive conventional ingredients such as roasted soya in broiler feed rations.

(C) 2021 International Formulae Group. All rights reserved.

Keywords: Curcuma longa, broilers, growth performance, carcass characteristics.

\section{INTRODUCTION}

L'aviculture est un moyen de subsistance le plus accessible à toutes les catégories sociales, en ce sens qu'il peut contribuer à la lutte contre la pauvreté, la sousalimentation, le sous-emploi (Grégoire et al., 2019). Elle constitue également la principale source de revenus pour plus de $86 \%$ des ménages surtout en milieu rural (FAO, 2019).

Pour satisfaire la demande croissante en produits avicoles, plusieurs contraintes sont à relever dont l'une des principales est l'accès aux aliments. En effet, les sources conventionnelles de protéines telles que les tourteaux de soja, d'arachide et la farine de poisson sont rares et donc coûteuses (Dahouda et al., 2009). Une alternative trouvée pour pallier le problème d'accès aux aliments est la valorisation des ressources alimentaires alternatives disponibles localement permettant d'améliorer la productivité des volailles tout en maitrisant les coûts des intrants (Ouédraogo, 2015). En effet, l'utilisation des aliments locaux non conventionnels dans l'alimentation animale apparaît comme une solution endogène (Dahouda et al., 2013). Les additifs alimentaires sont des composites nutritifs qui aident à améliorer l'efficacité de l'utilisation des aliments et par conséquent, à réduire le coût élevé de ces aliments (Zhang, 2009). A cet effet, l'incorporation du curcuma en substitution partielle ou totale à des ingrédients conventionnels comme le soja torréfié constitue une alternative intéressante pour assainir l'environnement et diminuer les charges liées à l'alimentation des poulets qui peuvent représenter $60 \%$ à $80 \%$ des charges des exploitations avicoles dans un système d'intégration agriculture - élevage comme indiqué par Hêdji et al. (2015). Le Curcuma en tant que promoteurs de croissance naturel peut être utilisé comme une alternative aux promoteurs de croissance artificiels courants tels que les antibiotiques (Demir et al., 2003). La curcumine est le composant bioactif indispensable à l'action biologique de Curcuma longa (Nouzarian et al., 2011). Un certain nombre d'expériences ont été menées pour évaluer l'effet de la curcumine sur les performances des poulets de chair et des poules pondeuses (Nouzarian et al., 2011) ainsi que (Ouédraogo et al., 2021). Le but de cette recherche était donc d'étudier l'effet de l'incorporation à des taux de $2 \%$ et $4 \%$ de poudre de Curcuma longa sur les paramètres de croissance des poulets.

\section{MATERIEL ET METHODES \\ Matériel \\ Site et période d'étude}

L'expérimentation a été réalisée dans un poulailler en matériaux définitifs, couvrant une superficie de $120 \mathrm{~m}^{2}$ situé à l'intérieur de la station de recherche de Farakoba à Bobo Dioulasso Région Ouest du Burkina Faso. L'étude s'est déroulée du 28 Décembre 2020 à fin Février 2021 soit une durée globale de deux (02) mois. Elle s'est déroulée dans l'ensemble, dans une période fraiche. 


\section{Animaux d'expérience}

Le matériel animal était composé de 120 poussins hybrides non sexés de 21 jours d'âge. Le poids moyen des poussins était de $370,40 \pm 26,22 \mathrm{~g}$. Ces poussins sont issus du croisement entre des coqs de race Bleu d'Hollande et des poules de race locale par incubation dans une couveuse électrique.

\section{Aliments}

Le curcuma utilisé a été produit à la station de recherche de Farakoba. Les autres ingrédients tels que le maïs, le son de mais, l'arachide, la farine de poisson le tourteau d'arachide, les acides aminés de synthèse ont été achetés dans le marché local (Figure 1 et Figure 2).

\section{Composition chimique}

Selon les travaux de Djoumessi et al. (2020), le Curcuma longa a une forte teneur en matière sèche $(84,1 \%)$, en matière organique (91,7\% MS) et en énergie digestible (3 628 $\mathrm{kcal} / \mathrm{kg} \mathrm{MS})$, protéine brute $(7,5 \% \mathrm{MS})$, Cendre $(8,3 \% \mathrm{MS})$, cellulose brute $(4,2 \% \mathrm{MS})$ et lipide (3,8\% MS).

\section{Rations alimentaires}

Pour la période de croissance-finition, trois régimes expérimentaux (R0, R1, R2) ayant strictement les mêmes valeurs nutritionnelles de croissance-finition ont été utilisés. Une ration témoin R0 sans additif a été fabriquée et deux autres rations formulées en incorporant à cette ration de base respectivement $2 \%, 4 \%$ de la poudre de curcuma longa (Tableau 1).

\section{Méthodologies}

\section{Production de la poudre de Curcuma}

Après leur récolte, les rhizomes ont été nettoyés pour éliminer les odeurs de terre dans laquelle ils ont été cultivés. Ensuite, ils ont été coupés en petits morceaux et séchés pendant une semaine environ à la lumière du soleil pour éliminer la teneur en humidité. Après séchage, le curcuma a été broyé finement et tamisé à travers un tamis de $1 \mathrm{~mm}$ pour former une poudre qui a été incorporée dans les régimes expérimentaux.

\section{Dispositif expérimental}

A l'âge de 21 jours, les 120 poussins ont été répartis de façon aléatoire en trois lots de 40 sujets correspondant aux trois traitements alimentaires R0, R1 et R2. Chaque lot a été subdivisé (de manière aléatoire) en deux souslots de 20 sujets correspondant à deux répétitions (A et $\mathrm{B}$ ) (Figure 3 ). Les sujets de chaque lot ont été par la suite identifiés grâce à des bagues en plastique placés sur l'aile droite.

\section{Programme de prophylaxie}

Avant l'installation des poussins, le poulailler a été nettoyé, désinfecté avec de la chaux et un vide sanitaire de deux semaines a été observé. Le protocole de prophylaxie médicale appliqué depuis l'éclosion des poussins jusqu'à la fin de l'expérience est rapporté dans le Tableau 2.

\section{Collecte des données et calcul des paramètres zootechniques de l'étude}

Les poids des oiseaux sont notés de façon hebdomadaire et les quantités d'aliments consommés (servis et refus) sont relevées par pesée quotidienne puis les carcasses pesées à l'abattage. Ce processus permet de calculer des paramètres comme la consommation alimentaire, la croissance pondérale, l'Indice de Consommation (IC) le taux de mortalité, le rendement carcasse.

\section{Traitement et analyses statistiques des données}

Les données ont été saisies et traités à l'aide du tableur Microsoft Office Excel. 2010. Ce même outil a été utilisé pour construire les tableaux et les graphiques. Les données ont été par la suite soumises à une analyse de variance (ANOVA) au seuil de 5\% selon le modèle de Fisher grâce au logiciel. R 3.5.1. En cas de différence significative, le test de comparaison multiple de Tukey HSD a été utilisé pour la séparation des moyennes. 


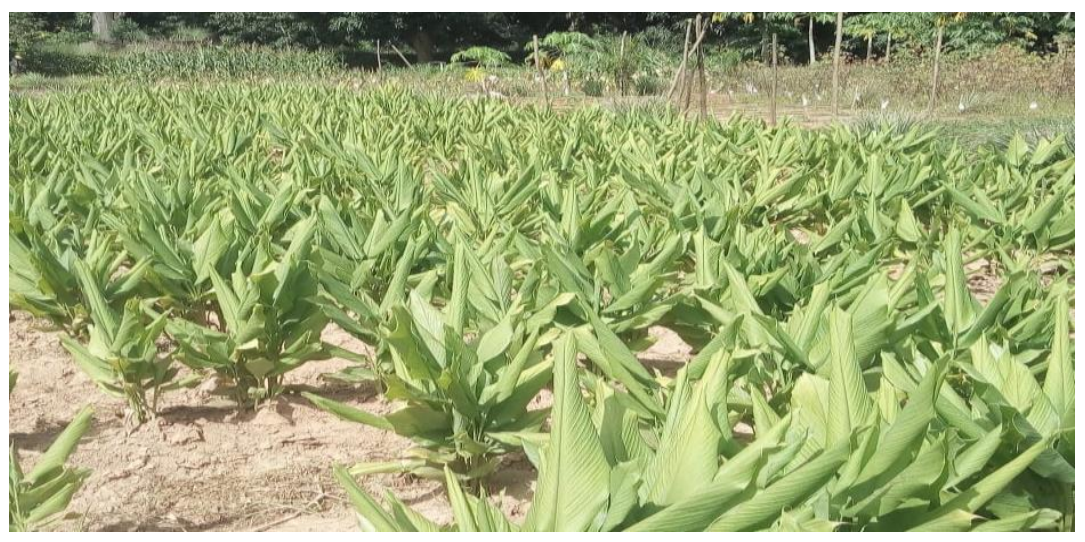

Figure 1 : Champ de Curcuma longa à la station de recherche de Farakoba (Bansé,2021).
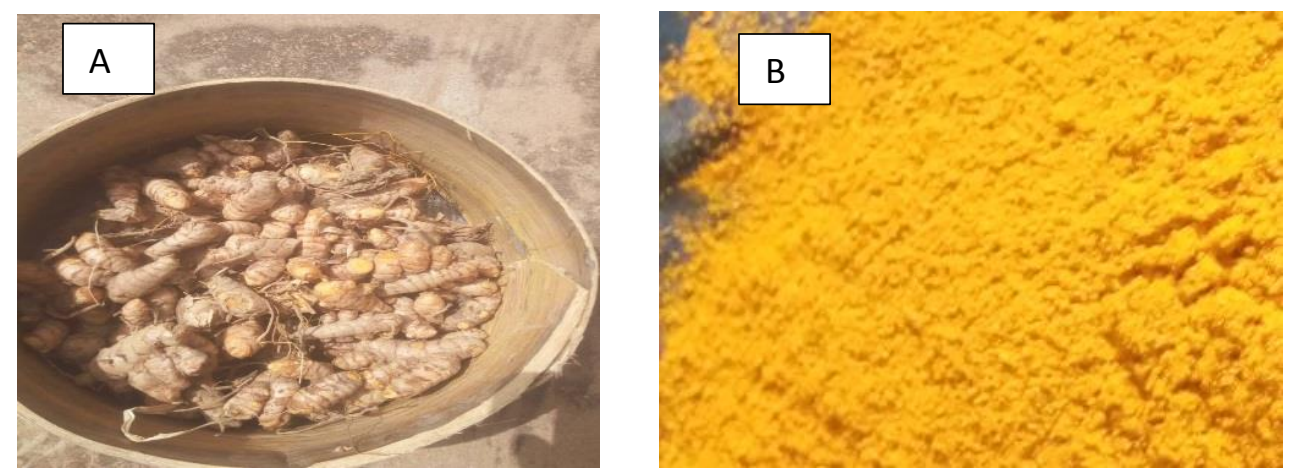

Figure 2 : Curcuma longa : Rhizome (A) et Poudre de rhizome (B) (Bansé,2021).

Tableau 1 : Composition centésimale (\%) des régimes de croissance - finition.

\begin{tabular}{lccc}
\hline \multirow{2}{*}{ Ingrédients } & \multicolumn{3}{c}{ Rations } \\
\cline { 2 - 4 } & TémoinR0 (0\%) & $\mathbf{R 1 ~ ( 2 \% )}$ & $\mathbf{R 2 ~ ( 4 \% )}$ \\
\hline Mais & 58 & 58 & 58 \\
\hline Son de blé & 19 & 19 & 19 \\
\hline Soja torréfié & 15 & 13 & 11 \\
\hline Poudre de Curcuma & 0 & 2 & 4 \\
\hline Farine de poisson & 5 & 5 & 2,5 \\
\hline CMV & 2,5 & 2,5 & 0,5 \\
\hline NaCl & 0,5 & 0,5 & 100 \\
\hline Total & 100 & 100 & 5 \\
\hline
\end{tabular}




\begin{tabular}{ccccccc}
\hline Blocs & \multicolumn{3}{c}{ Répétition A } & \multicolumn{3}{c}{ Répétition B } \\
\hline Traitements & R0 & R1 & R2 & R0 & R1 & R2 \\
\hline Nombre de sujet & 20 & 20 & 20 & 20 & 20 & 20 \\
\hline
\end{tabular}

Figure 3 : Schéma du dispositif expérimental.

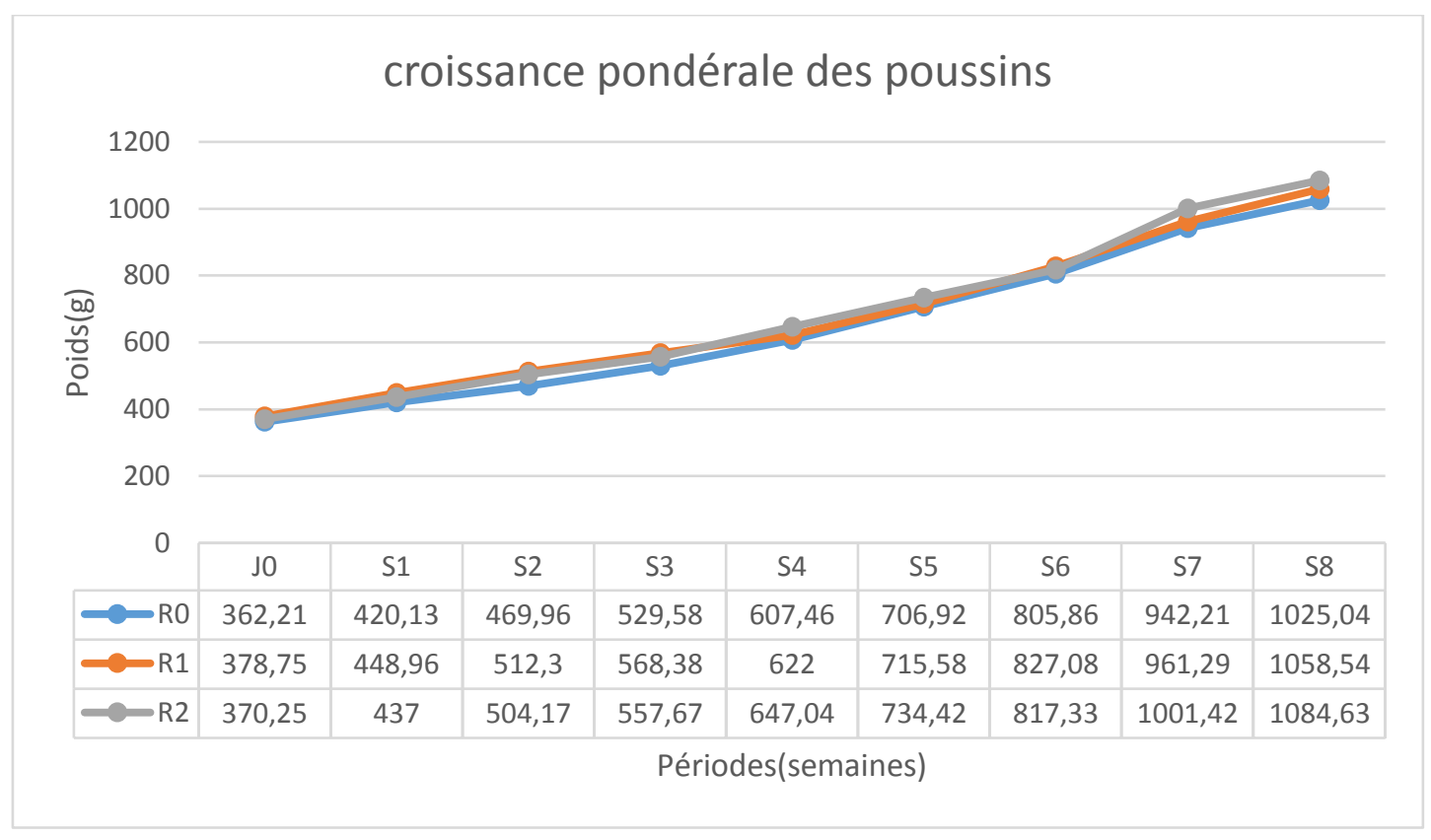

Figure 4 : Evolution pondérale des poussins.

Tableau 2 : Prophylaxie médicale appliquée.

\begin{tabular}{llll}
\hline Age (jour) & Actes & Produits utilisés \\
\hline 1 & $\begin{array}{l}\text { Vaccination contre les maladies de } \\
\text { Newcastle }\end{array}$ & HB1 (Trempage de bec, boisson) \\
\hline $1,2,3,4$ & Administration antistress et antibiotique & COLITERRAVET \\
\hline 9 & Vaccination contre la maladie de Gumboro & $\begin{array}{l}\text { Hypragumboro-CH80 (trempage et eau de } \\
\text { boisson) }\end{array}$ \\
\hline $9,10,11$ & Administration anti-stress & COLITETRAVET \\
\hline $\begin{array}{l}\text { 17, 18, } \\
19,20\end{array}$ & Administration d'anticoccidiens & Amprolium 20\% \\
\hline 21 & $\begin{array}{l}\text { Rappel vaccination contre la maladie de } \\
\text { Gumboro et Newcastle }\end{array}$ & $\begin{array}{l}\text { Hypragumboro-CH8 (trempage et eau de } \\
\text { boisson) }\end{array}$ \\
\hline $21,22,23$ & Administration anti-stress & Néoxyvital \\
\hline 28,56 & $\begin{array}{l}\text { Administration d'antiparasitaires et de } \\
\text { vitamines }\end{array}$ & $\begin{array}{l}\text { Citrate de pipérazine, Albendazole plus } \\
\text { vitamines }\end{array}$ \\
\hline
\end{tabular}




\section{RÉSULTATS}

\section{Analyses bromatologiques des aliments}

Les résultats de l'analyse bromatologique des différentes rations sont consignés dans le (Tableau 3). On constate que les trois rations (R0, R1 et R2) ont à peu près les mêmes teneurs en Matières Azotées Totales (MAT), la ration témoin (R0) est plus riche en matière grasse $(\mathrm{MG})$ que les rations $\mathrm{R} 1$ et $\mathrm{R} 2$. Par contre, les rations R1 et R2 ont une teneur plus élevée en cellulose brute (CB) que la ration R0. Les analyses nutritionnelles effectuées au laboratoire du centre de recherche abritant l'essai concernaient la détermination de la matière sèche (MS), la matière protéine brute, la cellulose brute (CB), les cendres, minérale, la matière grasse (MG). Les rations expérimentales sont isoénergétiques et iso-protéiques de type croissance-finition pour poulets de chair. (Tableau 3).

\section{Effet de l'incorporation sur les poids vifs}

L'effet de l'incorporation de la poudre de Curcuma longa dans les différentes rations sur l'évolution des poids vifs des animaux en fonction du temps est présenté sur la Figure 4. L'évolution pondérale des poussins en fonction des taux d'incorporation présente des variations non significatives entre les lots de S1à S8. A la fin de l'essai, c'est-à-dire à 8 semaines d'âge, on note une différence non significative au seuil de 5\% entre les traitements $(\mathrm{P}=0,719), \quad$ On constate que l'incorporation n'a pas amélioré significativement les poids vifs des sujets par rapport au témoin avec $\mathrm{R} 0(1025,04 \pm 287,55 \mathrm{~g})$, R1 $(1058,54 \pm 235,12 \mathrm{~g})$ et R2 $(1084,63 \pm 236,47$ g). L'évolution des poids vifs des animaux en fonction du temps est présentée sur la Figure 4.

\section{Effet de l'incorporation sur les consommations alimentaires}

Les consommations alimentaires obtenues chez les sujets des différents traitements alimentaires sont consignées dans le Tableau 4.

Le taux d'incorporation de la poudre de Curcuma longa a induit un effet non significatif au seuil de $5 \%(\mathrm{p}>0,05)$ sur la consommation alimentaire quelle que soit la phase d'étude. En effet, sur la période (S1-S2) de l'expérience, la consommation alimentaire a connu une variation significative d'un traitement à un autre $(\mathrm{P}<0,05)$. Si on considère toute la durée de l'expérience $\left(1^{\mathrm{è}}\right.$ à la $8^{\text {è }}$ semaine d'âge) la consommation alimentaire a connu globalement des variations non significatives $(\mathrm{P}=0,063)$ d'un traitement à un autre avec R0 $\quad(55,54 \pm 13,29 \mathrm{~g}), \quad \mathrm{R} 1$ $(54,72 \pm 11,3 \mathrm{~g})$ et $\mathrm{R} 2(51,8 \pm 12,67 \mathrm{~g})$

\section{Effet de l'incorporation sur les Gains Moyens Quotidiens(GMQ)}

Les GMQ obtenus des sujets des différents traitements alimentaires sont consignés dans le Tableau5.

Sur toute la période de l'essai [S1-S8], les GMQ présentent des différences non significatives $(P=0,474)$. Les résultats donnent R0 $(11,83 \pm 7,33 \mathrm{~g} / \mathrm{j}), \mathrm{R} 1(12,40 \pm 7,17 \mathrm{~g} / \mathrm{j})$, puis R2 (12,75 $\pm 8,02 \mathrm{~g} / \mathrm{j}) \quad$ (Tableau 5). D'une manière générale, les poulets nourris avec les régimes à 2 et $4 \%$ de curcuma présentent les meilleurs GMQ sur l'ensemble de la période même si les différences restent non significatives $(p>0,05)$.

\section{Effet sur l'Indice de Consommation (IC)}

Le Tableau 6 présente les résultats des indices de consommation alimentaire des poulets. Les indices de consommation alimentaire obtenus par traitement pendant toute l'expérimentation sont de R0 $(4,96 \pm 1,18) ; \mathrm{R} 1(4,83 \pm 1,23)$ et R2 $(4,39 \pm 1,15)$. L'incorporation de Curcuma dans la ration n'a engendré aucun effet néfaste significatif (Tableau 6) sur les indices de consommation alimentaire (IC) chez les sujets des différents traitements par rapport au témoin pendant toute la durée de l'expérimentation. Globalement? On note une amélioration de l'indice de consommation avec des rations contenant la poudre de Curcuma longa par rapport au témoin. Notons que l'indice de consommation des sujets nourris aux rations incorporant le curcuma est plus faible par rapport à l'indice de consommation avec la ration témoin même si les différences restent non significatives ( $p>0,05)$. En effet, les analyses statistiques ont 
montré que ces résultats obtenus présentent des différences non significatives $(\mathrm{P}=0,614)$ d'un traitement à l'autre au seuil de $5 \%$ sur toute l'expérimentation.

\section{Effet sur les caractéristiques de la carcasse et des organes}

Les effets de l'incorporation de la poudre de curcuma sur les caractéristiques de la carcasse et des organes des poulets sont consignés dans le Tableau 7. L'incorporation a conduit à une amélioration non significative du poids carcasse à 56 jours d'âge chez les poulets nourris avec R1 et R2 comparativement au traitement témoin même si les différences restent non significatives $(\mathrm{p}>0,05)$. Les poids carcasses sont significativement plus élevés dans les deux premiers lots (R1 et R2) que dans le témoin (R0). L'incorporation n'a engendré aucun effet néfaste sur le rendement carcasse des sujets nourris à base de la poudre de curcuma (Tableau 7). L'incorporation a conduit à une amélioration de la qualité de la chair avec une réduction du taux de graisse abdominale des poulets (Figure 5).

\section{Effet sur la mortalité}

Les taux de mortalité chez les poulets durant l'essai sont présentés dans le Tableau 8.

Il ressort de ce tableau que l'incorporation de la poudre de curcuma dans la ration, n'a eu aucun effet néfaste sur la santé et la mortalité des oiseaux. La majorité des mortalités est survenue entre la première et la deuxième semaine d'âge surtout dans les périodes les plus froides. L'autopsie n'a pas révélé une lésion, signe d'une maladie. Le taux moyen de mortalité est de $4,33 \%$ pour l'ensemble des lots. Il faut noter cependant que les poulets nourris avec la ration R0 ont enregistré des mortalités importantes (Tableau 8).

Tableau 3 : Composition chimique des différents types d'aliments distribués.

\begin{tabular}{c|c|c|c}
\hline Rations & TémoinR0 $(\mathbf{0 \%})$ & R1 $\mathbf{2 \%})$ & R2 (4\%) \\
\hline Matière sèche (\%) & 91,31 & 91,22 & 91,58 \\
\hline Protéine brute (\%) & 21,78 & 21.58 & 21.41 \\
\hline Matière grasse (\%) & 3,81 & 3,15 & 3,08 \\
\hline Cellulose brute (\%) & 3,28 & 3,19 & 3,21 \\
\hline Cendres (\%) & 6,9 & 7,1 & 7,3 \\
\hline
\end{tabular}

Tableau 4 : Consommation alimentaire des sujets.

\begin{tabular}{cccccc}
\hline \multirow{2}{*}{ Période } & \multicolumn{1}{c}{ LOTS } & \multicolumn{2}{c}{ Analyses statistiques } \\
\cline { 2 - 6 } & $\mathbf{R 0}$ & $\mathbf{R} 1$ & $\mathbf{R 2}$ & $\mathbf{P r}(>\mathbf{F})$ & Sign \\
\hline$[\mathrm{S} 1-\mathrm{S} 2]$ & $45,01 \pm 7,69 \mathrm{a}$ & $47,61 \pm 7,66 \mathrm{ab}$ & $38,94 \pm 5,37 \mathrm{~b}$ & 0,000 & $\mathrm{~S}$ \\
\hline$[\mathrm{S} 3-\mathrm{S} 4]$ & $49,12 \pm 9,05 \mathrm{a}$ & $49,66 \pm 7,76 \mathrm{a}$ & $45,60 \pm 6,79 \mathrm{a}$ & 0,120 & $\mathrm{NS}$ \\
\hline$[\mathrm{S} 5-\mathrm{S} 6]$ & $55,01 \pm 7,48 \mathrm{a}$ & $64,41 \pm 7,11 \mathrm{a}$ & $57,68 \pm 9,72 \mathrm{a}$ & 0,288 & $\mathrm{NS}$ \\
\hline$[\mathrm{S} 7-\mathrm{S} 8]$ & $73,03 \pm 7,31 \mathrm{a}$ & $67,20 \pm 11,00 \mathrm{ab}$ & $65,00 \pm 7,94 \mathrm{~b}$ & 0,003 & $\mathrm{~S}$ \\
\hline$[\mathrm{S} 1-\mathrm{S} 8]$ & $55,54 \pm 13,29 \mathrm{a}$ & $54,72 \pm 11,36 \mathrm{a}$ & $51,8 \pm 12,67 \mathrm{a}$ & 0,063 & $\mathrm{NS}$ \\
\hline Les valeurs de la même ligne, indicées de lettres différentes sont significativement différentes au seuil de $5 \%(p<0,05)$.
\end{tabular}


Tableau 5 : Évolution des Gains Moyens Quotidien des sujets.

\begin{tabular}{cccccc}
\hline \multirow{2}{*}{ Période } & \multicolumn{3}{c}{ LOTS } & \multicolumn{2}{c}{ Analyses statistiques } \\
\cline { 2 - 5 } & $\mathbf{R 0}$ & $\mathbf{R 1}$ & $\mathbf{R 2}$ & $\mathbf{P r}(>\mathbf{F})$ & Sign \\
\hline$[\mathrm{S} 1-\mathrm{S} 2]$ & $7,70 \pm 4,33$ & $9,54 \pm 3,77$ & $9,57 \pm 4,00$ & 0,143 & $\mathrm{NS}$ \\
\hline$[\mathrm{S} 3-\mathrm{S} 4]$ & $9,82 \pm 6,56$ & $7,84 \pm 5,27$ & $10,21 \pm 6,16$ & 0,122 & $\mathrm{NS}$ \\
\hline$[\mathrm{S} 5-\mathrm{S} 6]$ & $14,17 \pm 5,14$ & $14,65 \pm 7,66$ & $12,16 \pm 7,24$ & 0,166 & $\mathrm{NS}$ \\
\hline$[\mathrm{S} 7-\mathrm{S} 8]$ & $15,65 \pm 7,29$ & $16,53 \pm 7,50$ & $19,09 \pm 9,82$ & 0,111 & $\mathrm{NS}$ \\
\hline$[\mathrm{S} 1-\mathrm{S} 8]$ & $11,83 \pm 7,33$ & $12,40 \pm 7,17$ & $12,75 \pm 8,02$ & 0,474 & $\mathrm{NS}$ \\
\hline
\end{tabular}

Tableau 6 : Évolution des Indices de Consommation.

\begin{tabular}{cccccc}
\hline \multirow{2}{*}{ Période } & \multicolumn{2}{c}{ LOTS } & \multicolumn{2}{c}{ Analyses statistiques } \\
\cline { 2 - 5 } & $\mathbf{R 0}$ & $\mathbf{R 1}$ & $\mathbf{R 2}$ & $\mathbf{P r}(>\mathbf{F})$ & Sign \\
\hline$[\mathrm{S} 1-\mathrm{S} 2]$ & $5,92 \pm 1,19$ & $5,02 \pm 0,63$ & $4,07 \pm 0,42$ & 0,226 & $\mathrm{NS}$ \\
\hline$[\mathrm{S} 3-\mathrm{S} 4]$ & $5,04 \pm 0,35$ & $6,35 \pm 0,66$ & $4,76 \pm 1,65$ & 0,389 & $\mathrm{NS}$ \\
\hline$[\mathrm{S} 5-\mathrm{S} 6]$ & $3,88 \pm 0,28$ & $3,73 \pm 0,21$ & $4,76 \pm 0,69$ & 0,185 & $\mathrm{NS}$ \\
\hline$[\mathrm{S} 7-\mathrm{S} 8]$ & $5,01 \pm 2,00$ & $4,22 \pm 1,37$ & $3,99 \pm 1,18$ & 0,857 & $\mathrm{NS}$ \\
\hline$[\mathrm{S} 1-\mathrm{S} 8]$ & $4,96 \pm 1,18$ & $4,83 \pm 1,23$ & $4,39 \pm 1,15$ & 0,614 & $\mathrm{NS}$ \\
\hline
\end{tabular}

Tableau 7 : Caractéristiques de la carcasse des différents lots de poulets.

\begin{tabular}{cccccc}
\hline \multirow{2}{*}{ Paramètres } & $\mathbf{R}$ Lots & & \\
\cline { 2 - 5 } & $\mathbf{R}(\mathbf{0 \%})$ & $\mathbf{R 1}(\mathbf{2 \%})$ & $\mathbf{R 2} \mathbf{( 4 \% )}$ & $\mathbf{P r}(>\mathbf{F})$ & Sign \\
\hline Poids vifs & $1025,04 \pm 287,55$ & $1058,54 \pm 235,12$ & $1084,63 \pm 236,47$ & 0,053 & NS \\
\hline Poids carcasse & $810,04 \pm 0,71$ & $843,25 \pm 34,64$ & $869,25 \pm 54,42$ & 0,062 & NS \\
\hline Rendement carcasse & $79,025 \pm 1,35$ & $79,66 \pm 1,26$ & $80,14 \pm 1,50$ & 0,058 & NS \\
\hline Graisses abdominales & Trace & Trace & Trace & & NS \\
\hline
\end{tabular}

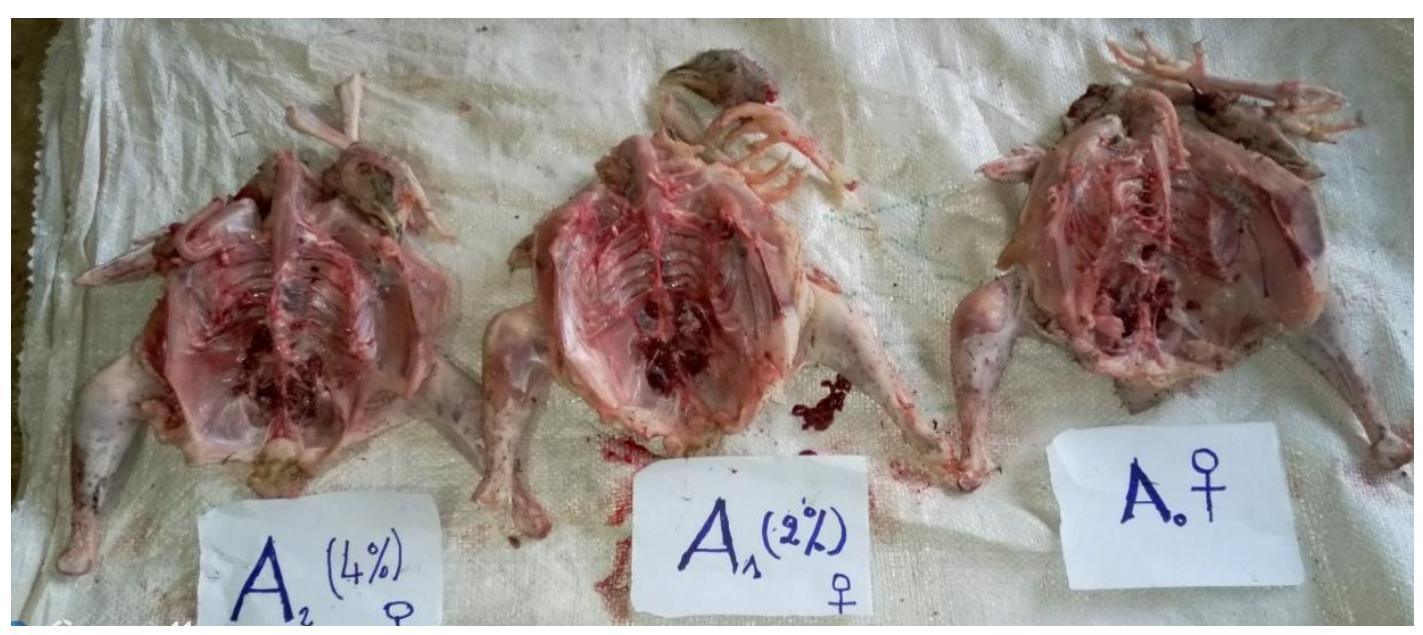

Figure 5 : Absence de graisses abdominales à l'abattage à 56 jours. 
Tableau 8 : Effet de l'incorporation des graines d'oseille sur la mortalité des poulets.

\begin{tabular}{ccccc}
\hline Paramètres & R0 & R1 & R2 & Moyenne \\
\hline 1 à 2 semaines & 5,00 & 3,00 & 2,00 & 3,33 \\
\hline 3 à 5 semaines & 1,00 & 1,00 & 1,00 & 1,00 \\
\hline 6 à 8 semaines & 0,00 & 0,00 & 0,00 & 0,00 \\
\hline 1 à 8 semaines & 6,00 & 4,00 & 3,00 & 4,33 \\
\hline
\end{tabular}

\section{DISCUSSION}

Effet de l'incorporation de la poudre de Curcuma longa sur les performances de croissance

On constate dans les conditions de notre étude que l'incorporation a entrainé une amélioration non significative sur les poids vifs et les caractéristiques de la carcasse des sujets par rapport au témoin. Ceci est contraire aux nombreux travaux comme ceux de Kumari et al. (2007) qui ont rapporté que la supplémentation en farine de curcuma à raison de $1,0 \mathrm{~g} / \mathrm{kg}$ améliorait les performances de croissance des poulets de chair âgés de 42 jours. De même Durrani et al. (2006) ont constaté que la supplémentation de 5,0 g / kg de farine de curcuma dans les régimes entraînait une amélioration significative du gain de poids corporel et de l'efficacité alimentaire sans aucun effet néfaste sur la mortalité. Dans la même lancée Ouédraogo et al. (2021) ont montré que l'incorporation du curcuma en tant qu'additif alimentaire a amélioré les performances de croissance, la consommation alimentaire, l'efficacité alimentaire et a entrainé la réduction du taux de graisse abdominale des poulets. L'amélioration de la performance de croissance due à la supplémentation en farine de curcuma dans toutes ces études pourrait s'expliquer par le fait que curcuma possède des activités antimicrobiennes, antifongiques et antioxydantes qui peuvent améliorer l'utilisation des nutriments alimentaires par les oiseaux (Radwan et al., 2008). L'amélioration des performances de croissance due à l'incorporation de la poudre de curcuma chez les poulets de chair pourrait être attribuée à l'amélioration du système digestif du poulet. En effet, Rajput et al. (2012) ont montré que la supplémentation alimentaire en curcumine pure à raison de $0,2 \mathrm{~g} / \mathrm{kg}$ dans un régime à base de maïs-soja augmentait la longueur et la largeur des villosités dans le duodénum, le jéjunum et la caeca de poulets de chair âgés de 42 jours. Cependant, nos résultats ne sont pas en accord avec ceux de certains auteurs qui n'ont pas trouvé d'effets bénéfiques sur la supplémentation alimentaire avec le curcuma à raison de 0,5 g / kg (Akbarian et al., 2012), 1,0 $\mathrm{g} / \mathrm{kg}$ (Rahmatnejad et al., 2009), 2,0 g / kg (Mehala et Moorthy, 2008), 5,0 g / kg (Gowda et al., 2008; Yarru et al., 2009), Abbas et al., 2010), soit 30,0 g / kg (Abbas et al., 2010). De même les résultats de Dingfa et al. (2015) ont révélé qu'un régime supplémenté en curcuma n'avait aucun effet significatif $(\mathrm{P}>0,05)$ sur le poids corporel, bien que les oiseaux nourris avec un régime avec du curcuma à 100 et 200 $\mathrm{mg} / \mathrm{kg}$ aient eu des poids vifs moyens plus élevés par rapport au témoin de 9 à 12 semaines. Nos résultats sont similaires à ceux d'Emadi (2006) qui a rapporté qu'une supplémentation alimentaire de 2,5,5 et 7,5 g / 
$\mathrm{kg}$ de curcuma n'avait aucun effet sur le gain de poids des poulets de chair

\section{Caractéristiques des carcasses}

Les poids carcasses ont connu des différences non significatives pour les deux premiers lots (R1 et R2) ainsi que chez le témoin (R0). L'incorporation n'a engendré aucun effet néfaste sur le rendement carcasse. Ceci est en accord avec certains auteurs qui informent que la supplémentation alimentaire avec du Curcuma peut avoir des effets bénéfiques sur les caractéristiques de la carcasse des poulets de chair car elle contient des composés phytochimiques bénéfiques, comme la curcumine, la méthylcurcumine et d'autres composés actifs. Les résultats de cette étude sont en accord avec ceux de Kafi et al. (2017) qui indiquent que la supplémentation alimentaire en farine de curcumine à raison de $0,35 \mathrm{~g} / \mathrm{kg}$ a réduit la teneur en graisse abdominale, l'épaisseur de la graisse souscutanée, la largeur de la graisse intermusculaire et la graisse du foie des poulets mâles Wanjiang Yellow. Les dépôts de graisse dans la région abdominale des poulets de chair sont considérés comme des déchets dans la production avicole, car ils représentent une perte sur le marché et une acceptabilité réduite par les consommateurs. Les résultats de la présente étude ont indiqué que la supplémentation en Curcuma des aliments pour poulets de chair a le potentiel de réduire ce type de déchets en réduisant la teneur en graisse abdominale. Nouzarian et al. (2011) ont rapporté également que l'ajout de poudre de Curcuma $(3,3,6,6$ et $10 \mathrm{~g} / \mathrm{kg}$ de régime) réduisait considérablement le poids de la graisse abdominale des poulets de chair, par rapport au groupe témoin. Dans le même registre une étude de Kafi et al.(2017) a rapporté que le pourcentage de poids de graisse abdominale et hépatique ainsi que l'épaisseur de graisse sous-cutanée des poulets de chair
Arbor Acres et Wangjiang Yellow ont été réduits après une supplémentation de 0,25 à $0,35 \mathrm{~g} / \mathrm{kg}$ de curcumine. En utilisant un niveau de supplémentation plus élevé $(5,0 \mathrm{~g} / \mathrm{kg})$, Durrani et al. (2006) ont montré les effets bénéfiques de la supplémentation alimentaire au Curcuma pour réduire la teneur en matières grasses, augmenter la qualité de la carcasse. De même, Nouzarian et al. (2011) ont rapporté que l'ajout de poudre de Curcuma (3,3, 6,6 et $10 \mathrm{~g}$ $/ \mathrm{kg}$ de régime) réduisait considérablement le poids de la graisse abdominale des poulets de chair, par rapport au témoin. Mais leurs résultats sont contraires à ceux de Mehala et Moorthy (2008) qui n'ont observé aucun impact significatif de la poudre de Curcuma (10 g/kg de régime) sur le pourcentage de carcasses de poulets de chair élevés jusqu'à 42 jours.

\section{Effet de l'incorporation de la poudre de Curcuma longa sur les consommations alimentaires}

$\mathrm{Si}$ on considère toute la durée de l'expérience ( $1^{\text {è }}$ à la $8^{\text {è }}$ semaine d'âge) la consommation alimentaire a connu globalement des variations non significatives $(\mathrm{P}=0,063)$ d'un traitement à un autre avec $\mathrm{R} 0$ $(55,54 \pm 13,29 \mathrm{~g}), \mathrm{R} 1 \quad(54,72 \pm 11,3 \mathrm{~g})$ et R2 $(51,8 \pm 12,67 \mathrm{~g})$. Nos observations sont en désaccord avec celles de Durrani et al.(2006) qui ont constaté une consommation alimentaire significativement plus faible avec une supplémentation en Curcuma plus élevée dans le régime alimentaire des poulets de chair. Dans une même logique, Abbas et al. (2010) ont utilisé $1 \%, 2 \%$ et $3 \%$ d'inclusion de Curcuma comme promoteur de croissance et ont observé une meilleure prise alimentaire, un gain de poids.

Ainsi, nous pouvons conclure que l'utilisation à long terme du curcuma en poudre pourrait être un additif alimentaire utile 
car elle n'a pas induit d'effet délétère évident sur la santé des sujets.

\section{Effet de l'incorporation de la poudre de Curcuma sur l'indice de consommation(IC)}

L'indice de consommation des sujets nourris aux rations incorporant le Curcuma a révélé des différences non significatives par rapport à l'indice de consommation des rations témoin. Ce résultat ne concorde avec celui de Wang et al. (2016) qui indiquent qu'en raison de l'augmentation des enzymes telles que l'amylase, la trypsine, la chymotrypsine et la lipase, la conversion alimentaire pourrait être améliorée par les régimes alimentaires incorporant du curcuma chez les poules pondeuses. Contrairement toujours à nos résultats, une autre étude de Lagana et al. (2011) informe que l'indice de conversion a été réduit par l'incorporation de $2 \%$ de poudre de rhizome de Curcuma dans l'alimentation des poules pondeuses.

Cependant, Gumus et al. (2018) ont observé que l'ajout de $0,5 \%$ de curcuma augmentait la production et le poids des œufs, mais réduisait la conversion alimentaire par rapport au groupe témoin. La différence dans les réponses des poulets de chair rapportée dans ces études était due à la différence entre les régimes de base, les périodes d'élevage des poulets de chair ou les autres détails expérimentaux utilisés dans ces études, tels que la conception statistique, la supplémentation en doses et la race des oiseaux.

\section{Conclusion}

Le développement de l'élevage et l'amélioration de l'efficacité nutritionnelle des aliments obligent donc à avoir recours à l'emploi d'additifs alimentaires dont l'utilisation s'est généralisée en alimentation animale depuis de nombreuses années. Les résultats de la présente étude ont montré que l'incorporation du Curcuma en tant qu'additif alimentaire en substitution partielle au soja torréfié a entrainé des différences non significatives des performances de croissance, de la consommation alimentaire, de l'efficacité alimentaire et a entrainé la réduction du taux de graisse abdominale des poulets. Le Curcuma (Curcuma longa) peut être une alternative dans le système de production de volaille mais la connaissance de cet additif est encore limitée tant sur ses modes d'action que sur les aspects de son application. En effet, jusqu'à présent, peu de travaux ont été menés pour suggérer son mode d'action chez la volaille dans notre pays. Ces paramètres sans être significativement différents $(\mathrm{P}>0,05)$, ont montré que les meilleurs poids ainsi que les meilleurs rendements carcasse ont été obtenus avec les rations R1 et R2. L'étude a montré que l'incorporation du Curcuma à des taux de 2 et $4 \%$ pourrait réduire les charges en d'autres ingrédients comme le soja torréfié dans les rations alimentaires des volailles. Par ailleurs, pour optimiser l'efficacité de la supplémentation en Curcuma dans l'alimentation des volailles, les recherches futures devraient se concentrer davantage sur le mécanisme d'action, la dose optimale de supplémentation. Egalement une étude de rentabilité devrait être menée

\section{CONFLIT D'INTÉRÊTS}

Les auteurs déclarent qu'ils n'ont pas de conflit d'intérêts

\section{CONTRIBUTIONS DES AUTEURS}

$\mathrm{BO}$ et ZSJ ont contribué à l'élaboration et l'édition du protocole de recherche. BO a assuré la rédaction du manuscrit et a effectué la collecte, la saisie et l'analyse des données sous la supervision de ZSJ. SJ et ZSN ont produit et faciliter l'obtention de la poudre de rhizome de Curcuma longa. 


\section{REMERCIEMENTS}

Les auteurs adressent leurs sincères remerciements à Monsieur Ouédraogo Mahamadī, étudiant à l'Université Polytechnique de Bobo Dioulasso pour son soutien pendant le suivi de l'essai. Ils remerciements également Compaoré Nadège étudiante à l'Université Saint Thomsas d'Aquin (USTA) de Ouagadougou pour le suivi de l'essai aux côtés de Ouédraogo Mahamadī.

\section{REFERENCES}

Abbas RZ, Iqbal Z, Khan MN, Zafar MA, Zia MA. 2010. Anticoccidial Activity of Curcuma longa L. in Broilers. Brazilian Archives of Biology and Technology an International Journal, 53: 266-273. DOI: https://doi.org/10.1590/S151689132010000100008

Al-Jaleel RA. 2012. Use of Turmeric (Curcuma longa) on the Performance and Some Physiological Traits on the Broiler Diets. The Iraqi Journal of Veterinary Medicine, 36: 51-57. DOI: https://doi.org/10.30539/iraqijvm.v36i1. 548

Abbas RZ, Iqbal Z, Khan MN, Zafar MA, Zia MA. 2010. Anticoccidial Activity of Curcuma longa L. in Broilers. Brazilian Archives of Biology and Technology an International Journal, 53: 266-273. DOI: https://doi.org/10.1590/S151689132010000100008

Al-Jaleel RA. 2012. Use of Turmeric (Curcuma longa) on the Performance and Some Physiological Traits on the Broiler Diets. The Iraqi Journal of Veterinary Medicine, 36: 51-57. DOI: https://doi.org/10.30539/iraqijvm.v36i1. 548

Al-Sultan SI. 2003. The effect of Curcuma longa (turmeric) on overall performance of broiler chickens. Int. J. Poult. Sci., 2:
351-353.

DOI:

10.3923/ijps.2003.351.353

Akbarian A, Golian A, Kermanshahi H, Gilani A, Moradi S. 2012. Influence of turmeric rhizome and black pepper on blood constituents and performance of broiler chickens. Afr. J. Biotechnol., 11: 86068611. DOI: $10.5897 / A J B 11.3318$

Bansé O, Jacob S, Zara SN, Sibiri JZ. 2021. Effet de l'utilisation de la poudre de rhizome de curcuma comme additif alimentaire sur les performances de croissance et les caractéristiques de la carcasse des poulets de chair. Journal of Applied Biosciences, 163: 16820 - 16833. DOI:

https://doi.org/10.35759/JABs.163.3

Dahouda M, Toléba SS, Senou M, Youssao AKI, Hambuckers A, Hornick JL. 2009. Les ressources alimentaires nonconventionnelles utilisables pour la production aviaire en Afrique : valeurs nutritionnelles et contraintes. Ann. Méd. Vét., 153: 5-21.

Dahouda M, Adjolohoun S, Senou M, Toleba SS, Abou M, Vidjannagni DS, Kpodékon M, YoussaoAKI. 2013. Effets des aliments contenant des folioles de Moringa oleifera Lamet des aliments commerciaux sur les performances de croissance des lapins (Oryctolagus cuniculus). International Journal of Biological and Chemical Sciences, 7(5): 1838-1852. DOI: 10.4314/ijbcs.v7i5.5

Demir E, Sarica S, Ozcan MA, Suicmez M. 2003. The use of natural feed additives as alternatives for an antibiotic growth promoter in broiler diets. British Poultry Science, 44: 44-45. DOI: https://doi.org/10.1080/713655288

Durrani FR, Ismail M, Sultan A, Suhail SM, Chand N, Durrani Z. 2006. Effect of different levels of feed added turmeric 
(Curcuma longa) on the performance of broiler chicks. J. Agric. Biol. Sci., 1: 9-11.

Dingfa W, Huifang H, Luli Z, Wei L, Hanlin Z, Guanyu H, Jia L, Lin H. 2015. Effects of Dietary Supplementation with Turmeric Rhizome Extract on Growth Performance, Carcass Characteristics, Antioxidant Capability, and Meat Quality of Wenchang Broiler Chickens. Italian Journal of Animal Science, 14: 3. DOI: 10.4081/ijas.2015.3870

Djoumessi TFG, Tendonkeng F, Miégoué E, Camara S, Fokom Wauffo D, Emalé C, Pamo TE. 2020. Effet de différents niveaux de Curcuma longa dans la ration sur les performances de reproduction et de croissance pré-sevrage des cochons d'Inde. Livestock Research for Rural Development, $\quad 32(6)$. http://www.lrrd.org/lrrd32/6/ftend32088. html

Emadi M, Kermanshahi H. 2006. Effect of turmeric rhizome powder on performance and carcass characteristics of broiler chickens. Int. J. Poultry Sci., 5: 10691072. DOI: 10.3923/ijps.2006.1069.1072

FAO. 2019. Le devenir de l'élevage au Burkina

Faso: Défis et opportunités face aux incertitudes, 56p. http://www.fao.org

Grégoire N, Walter O, Ayao M, Simplice BA. 2019. Analyse de l'importance socioéconomique de l'aviculture familiale dans le Département de Salemata au Sénégal. International Journal of Biological Chemical Sciences, 13(7): 3131-3143. DOI: $10.4314 /$ ijbcs.v13i7.13

Gowda NKS, Ledouxa DR, Rottinghausa GE, Bermudeza AJ, Chena YC. 2009. Antioxidant efficacy of curcuminoids from turmeric (Curcuma longa L.) powder in broiler chickens fed diets containing aflatoxin $\mathrm{B}_{1} . \mathrm{Br}$. J. Nutr., 102: 1629-1634.

DOI: $10.1017 /$ S0007114509990869
Gumus H, Oguz MN, Bugdayci KE, Oguz FK. 2018. Effects of Sumac and Turmeric as Feed Additives on Performance, Egg Quality Trait, and Blood Parameters of Laying Hens. Revista Brasileira de Zootecnia, 47: 11-22. DOI: https://doi.org/10.1590/rbz4720170114

Hêdji CC, Marcel H, Frédéric H, Emile F. 2015. Assainissement de l'environnement par la valorisation des ressources non conventionnelles en alimentation de porcs en croissance. International Journal of Biological Chemical Sciences, 9(4): 1929-1936 DOI: $10.4314 /$ ijbcs.v9i4.18

Kafi A, Uddin MN, Uddin MJ, Khan MMH, Haque ME. 2017. Effect of Dietary Supplementation of Turmeric (Curcuma longa), Ginger (Zingiber officinale) and their Combination as Feed Additives on Feed Intake, Growth Performance and Economics of Broiler. International Journal of Poultry Science, 16: 257-265. DOI: 10.3923/ijps.2017.257.265

Kumari P, Gupta MK, Ranjan R, Singh KK, Yadava R. 2007. Curcuma longa as feed additive in broiler birds and its pathophysiological effects. Indian J. Exp. Biol., 45: 272-277. https://www.ajol.info

Lagana C, Pizzolante CC, Saldanha E, and Moraes JE. 2011. Turmeric Root and Annato Seed in Second-Cycle Layer Diets: Performance and Egg Quality. Brazilian Poultry Science, 13: 171-176. DOI: $\quad$ https://doi.org/10.1590/S1516635X2011000300002

Mehala C, Moorthy M. 2008. Production performance of broilers fed with Aloe vera and Curcuma longa (Turmeric). Int. J. Poult. Sci., 7: 852-856. DOI: 10.3923/ijps.2008.852.856

Moorthy MS, Ravi M, Ravikuma K, Viswanathan, Edwin SC. 2009. Ginger, pepper and curry leaf powder as feed 
additives in broiler diet. Int. J. Poult. Sci., 8: 779-782. DOI: 10.3923/ijps.2009.779.782

Nouzarian R, Tabeidian SA. Toghyani M, Ghalamkari G, Toghyani M. 2011. Effect of turmeric powder on performance, carcass traits, humoral immune responses, and serum metabolites in broiler chickens. J. Anim. Feed Sci., 20: 389-400.

DOI: https://doi.org/10.22358/jafs/66194/2011

Ouédraogo B, Balé B, Zoundi SJ, Sawadogo L. 2015. Caractéristiques de l'aviculture villageoise et influence des techniques d'amélioration sur ses performances zootechniques dans la province du Sourou, région Nord-Ouest Burkinabè. Int. J. Biol. Chem. Sci., 9(3): 1528-1543. DOI: 10.4314/ijbcs.v9i3.34

Radwan LN, Hassan RA, Qota EM, Fayek HM. 2008. Effect of Natural Antioxidant on Oxidative Stability of Eggs and Productive and Reproductive Performance of Laying Hens. International Journal of Poultry Science, 7: 134-150. DOI: https://doi.org/10.3923/ijps.2008.134.15 0

Rahmatnejad E, Roshanfekr H, Ashayerizadeh O, Mamooee M, Ashayerizadeh A. 2009. Evaluation the effect of several nonantibiotic additives on growth performance of broiler chickens. J. Anim. Vet. Adv., 8: 1670-1673.

Rajput N, Muhammah N, Yan R, Zhong X, Wang T. 2012. Effect of dietary supplementation of curcumin on growth performance, intestinal morphology and nutrients utilization of broiler chicks. $J$ Poult. Sci., 50(1): 44-52. DOI: 10.2141/jpsa.0120065

Sugiharto I, Widiastuti E, Prabowo NS. 2011. Effect of turmeric extract on blood parameters, feed efficiency and abdominal fat content in broilers. $J$. Indonesian Trop. Anim. Agric., 36: 21-26. DOI: $10.14710 /$ jitaa.36.1.21-26

Wang D. 2016. Effects of Dietary Supplementation with Turmeric rhizome Extract on Growth Performance, Carcass Characteristics, Antioxidant Capability, and Meat Quality of Wenchang Broiler Chickens. Italian Journal Animal Science, 14: 344-349. DOI: https://doi.org/10.4081/ijas.2015.3870

Yarru LP, Settivari RS, Gowda NKS, Antoniou E, Ledoux DR, Rottinghaus GE. 2009. Effects of turmeric (Curcuma longa) on the expression of hepatic genes associated with biotransformation, antioxidant, andimmune systems in broiler chicks fed aflatoxin. J. Poult Sci., 88: 2620-2627. DOI: $10.3382 /$ ps.2009-00204

Zhang GF, Yang ZB, Wang Y, Yang WR, Jiang SZ, Gai GS. 2009. Effects of ginger root (Zingiber officinale) processed to different particle sizes on growth performance, antioxidant status and serum metabolites of broiler chickens. Poult. Sci., $\quad$ 88: 2159-2166. DOI: $10.3382 /$ ps.2009-00165 